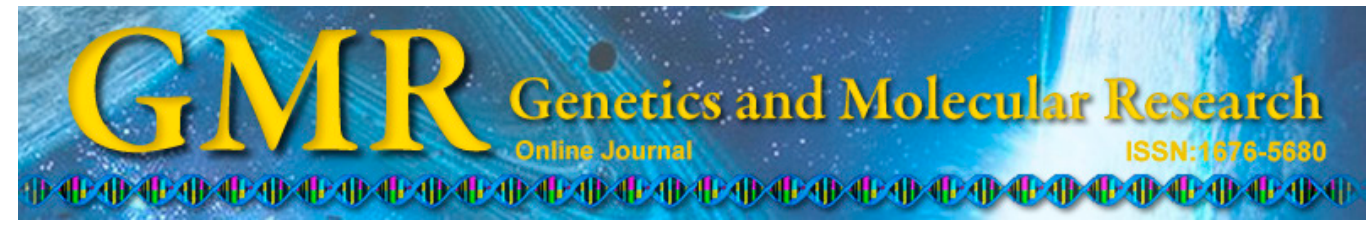

\title{
Differential expression of interleukin-12 p35 and 440 subunits in response to Aeromonas hydrophila and Aquareovirus infection in grass carp, Ctenopharyngodon idella
}

\author{
N.P. Pandit ${ }^{1,2}$, Y.B. Shen ${ }^{1}$, X.Y. Xu ${ }^{1}$, H.Y. Yu ${ }^{1}$, W.J. Wang ${ }^{1}$, R.Q. Wang ${ }^{3}$, \\ Y.F. Xuan ${ }^{3}$ and J.L. Li ${ }^{1,4}$ \\ ${ }^{1}$ Key Laboratory of Freshwater Fishery Germplasm Resources, \\ Ministry of Agriculture, Shanghai Ocean University, Shanghai, China \\ ${ }^{2}$ Institute of Agriculture and Animal Science, Paklihawa Campus, \\ Tribhuvan University, Rupendehi, Nepal \\ ${ }^{3}$ Key Laboratory of Conventional Freshwater Fish Breeding and Health \\ Culture Technology Germplasm Resources, Ministry of Agriculture, \\ Suzhou Wujiang Area Aquaculture Limited Company, Suzhou, China \\ ${ }^{4}$ E-Institute of Shanghai Universities, Shanghai Ocean University, Shanghai, \\ China \\ Corresponding author: J.L. Li \\ E-mail: j1li2009@126.com
}

Genet. Mol. Res. 14 (1): 1169-1183 (2015)

Received April 24, 2014

Accepted December 5, 2014

Published February 6, 2015

DOI http://dx.doi.org/10.4238/2015.February.6.20

ABSTRACT. The grass carp (Ctenopharyngodon idella) aquaculture industry in Asia is prone to bacterial and viral hemorrhagic diseases. Effective adjuvants for vaccine formulation are the need of the hour for control of these diseases and long-term sustainability of grass carp farming. In this study, the involvement of interleukin-12 (IL-12) from grass carp (gcIL-12) in anti-bacterial and anti-viral immune responses was demonstrated via expression profiles of gcIL-12 subunits in immune tissues of the fish, following infection by Aeromonas hydrophila and 
Aquareovirus. Additionally, cDNA of the gcIL-12 subunits, p35 and p40 was cloned and characterized. We found that most of the structurally and functionally important features of vertebrate orthologues were conserved in gcIL-12 subunits, p35 and p40, with some features specific to grass carp. High levels of gcIL-12 p35 expression in the brain and gills suggest that IL-12 plays an important role in neural and immune systems. High expression levels in the heart, blood, and immune-related tissues suggest an important role in circulation and the immune system as well. Infections by both, A. hydrophila and Aquareovirus stimulated the mRNA expression of gcIL-12 subunits, p35 and p40 in most immune tissues. Significant upregulation or downregulation of gcIL-12 subunits, p35 and p40 by bacterial and viral infection confirms their potential role in anti-bacterial and anti-viral immune responses in fish.

Key words: Grass carp; IL-12 p35; IL-12 p40; Aeromonas hydrophila; Aquareovirus; mRNA expression

\section{INTRODUCTION}

Grass carp (Ctenopharyngodon idella), is one of the most common fish commercially cultivated for food in Asia and is the highest contributor to the world's freshwater fish production (FAO, 2012). However, intensive culture practices have led to high incidence of disease in the fish, resulting in heavy losses to the aquaculture industry each year. Aeromonas hydrophila and Aquareovirus causing bacterial hemorrhagic septicemia and viral hemorrhagic disease, respectively in grass carp are the most destructive pathogens affecting grass carp farms (Liu et al., 2010; Wang et al., 2012). Infectious diseases caused by these pathogens result in more than $85 \%$ mortality in fingerling and yearling grass carp, leading to huge economic losses (Jiang, 2009; Jang et al., 2010). Preventing these infectious diseases is still a great challenge because of environmental concerns associated with chemical treatments and the emergence of multiple resistances to antibiotics (Vivekanandhan et al., 2002). Thus, extensive research on the molecular genetics of grass carp and a deeper understanding of their immune defense mechanisms are required to develop more efficient management strategies for disease control.

Interleukin-12 (IL-12) is the primary pro-inflammatory cytokine that stimulates interferon-gamma (IFN- $\gamma$ ) production by T cells and natural killer (NK) cells favoring the differentiation of $\mathrm{Th}_{1}$ cells and forming a link between innate resistance and adaptive immunity (Trinchieri, 2003). IL-12 is mainly produced by the activated antigen-presenting cells (APCs) and phagocytic cells, including monocytes/macrophages, neutrophils, dendritic cells, and activated B cells (Vignali and Kuchroo, 2012). In higher vertebrates, it plays a key role in the stimulation of cell-mediated immune response, as it promotes the differentiation of native $\mathrm{T}$ cells into $\mathrm{Th}_{1}$ phenotype in the presence of IL-2, while inhibiting the development of $\mathrm{Th}_{2}$ cells, mainly through its IFN- $\gamma$ production-stimulating activity (Gaucher and Chadee, 2001). In addition to immune defense mechanisms, IL-12 is also involved in the pathogenesis of $\mathrm{Th}_{1}$-mediated chronic inflammatory disorders in mammals (Adorini, 1999). Because of its immunoregulatory and anti-angiogenic activities, there has been an interest in exploring IL-12 as a candidate vaccine adjuvant and anti-cancer immunotherapeutic agent.

Biologically active IL-12 is a heterodimeric cytokine composed of two disulfide- 
linked subunits, p35 and p40, encoded by two different genes (Kobayashi et al., 1989), located on different chromosomes (Ebner et al., 2001); however, they are linked by an inter-chain disulfide bridge to form a functional molecule. Co-expression of p40 and p35 subunits in the same cell and secretion of the covalently linked heterodimer p70 are essential for biological activity (D'Andrea et al., 1992). The gene encoding p35 is constitutively expressed in different cell types and tissues, whereas that encoding p40 is more confined to APCs and neutrophils, and is strongly induced by intracellular bacteria and bacterial products that stimulate $\mathrm{Th}$ responses (Gubler et al., 1991). The p40 subunit is often expressed to a larger extent than the p35 subunit, and the p40 dimer binds to the IL-12 receptor on target cells (Ling et al., 1995). The expression and functions of IL-12 are mediated by a receptor composed of two chains, IL$12 \mathrm{R} \beta 1$ and IL-12Rß2 (Staerk and Constantinescu, 2012). Expression of both chains is essential for high-affinity binding and signal transduction. Although IL-12 has been widely studied in mammals, so far, it has been characterized in a few teleost species. In order to explore the role of IL-12 in the immune response and evaluate possible clinical usefulness, it is important to study the effects of this cytokine in different animal models.

To determine whether IL-12 is an active participant in specific bacterial and viral infections of freshwater fish, we cloned the genes encoding gcIL-12 subunits, p35 and p40 and measured their expression levels after infection by A. hydrophila and Aquareovirus. We observed that both gcIL-12 subunits actively participate in anti-Aeromonas and anti-Aquareovirus immune response. The findings from this study provide a basis to understand the basic mechanisms involved in adaptive immunity in grass carp and other teleost species.

\section{MATERIAL AND METHODS}

\section{Fish rearing, microbial infection, and sample preparation}

Adult and juvenile grass carps were obtained from the Nanhui Fish Farm in Shanghai and acclimatized at $26^{\circ} \mathrm{C}$ in 570-L aerated tanks for a week. For full-length cDNA cloning and tissue expression analysis, various tissues including the brain, gills, fins, skin, liver, trunk kidney, head kidney, heart, blood, spleen, and intestines were collected from 6 adult grass carps weighing approximately $2000 \mathrm{~g}$. All animal handling and experiments were conducted in accordance with the guidelines approved by the institutional animal care and use committee (IACUC) of Shanghai Ocean University.

Bacterial and viral challenge experiments were conducted as previously described by Pandit et al. (2013) with some modifications. Briefly, 72 juvenile fish weighing approximately $50 \mathrm{~g}$ were divided into 3 equal groups and cultured in separate 200-L tanks under similar conditions in a well-aerated system at $26^{\circ} \mathrm{C}$. Fish from the bacterial-challenge group were injected intraperitoneally with a suspension of live Aeromonas hydrophila S2 (obtained from the aquatic pathogen collection centre of the Ministry of Agriculture, China) at a dose of $1.0 \times 10^{6}$ cells in $100 \mu \mathrm{L}$ phosphate buffered saline (PBS) per fish. Similarly, fish from the viral-challenge group were injected intraperitoneally with a suspension of live Aquareovirus (GCRV-JX01) (obtained from the aquatic pathogen collection centre of the Ministry of Agriculture, China) at a dose of $1.0 \times 10^{6} \mathrm{TCID}_{50} / \mathrm{mL}$ in $100 \mu \mathrm{L}$ PBS per fish. In the control group, fish were injected with $100 \mu \mathrm{L}$ sterile PBS per fish. These pathogen doses were based on our preliminary experiment, in which we observed that these dose levels did not cause death but produced hemorrhage in the fish. Samples were collected from 6 fish at 4 h, 1 day, 3 days, and 
7 days post-injection from each group, respectively. Head kidney, spleen, gill, and intestine samples were collected from each fish for total RNA isolation.

Tissues were flash frozen in liquid nitrogen followed by storage at $-80^{\circ} \mathrm{C}$ until RNA extraction. Total RNA was isolated using TRIZOL Reagent (Invitrogen) following the manufacturer protocol. All RNA samples were treated with RNase-free DNase (TaKaRa, Japan), quantified using a Nanodrop 2000C spectrophotometer (Thermo Scientific, USA), and stored at $-80^{\circ} \mathrm{C}$ until use. RNA samples with a $260 / 280$ ratio between 1.90 and 2.10 and a 260/230 ratio between 2.00 and 2.50 were considered satisfactory and used in this study.

\section{Cloning of full-length gcIL-12 p35 and p40 cDNA by rapid amplification of cDNA ends (RACE) method}

The first strand of cDNA was synthesized from the total spleen mRNA using the PrimeScript high-fidelity reverse transcriptase polymerase chain reaction (RT-PCR) kit (Ta$\mathrm{KaRa}$, Japan). We previously constructed a cDNA library from C. idella spleen and kidney tissue (unpublished data), from which 523-bp and 850-bp fragments of IL-12 p35 and p40, respectively, were obtained. The short sequences were verified using gene-specific primers (Table 1) and used to clone full-length cDNA using the RACE method. The PCR program used for short sequence verification of gcIL-12 subunit $\mathrm{p} 35$ was: 1 cycle of $94^{\circ} \mathrm{C} / 3 \mathrm{~min} ; 30$ cycles of $94^{\circ} \mathrm{C} / 30 \mathrm{~s}, 52^{\circ} \mathrm{C} / 30 \mathrm{~s}, 72^{\circ} \mathrm{C} / 2 \mathrm{~min} ; 1$ cycle of $72^{\circ} \mathrm{C} / 10 \mathrm{~min}$. The PCR program used for gcIL-12 subunit p40 was: 1 cycle of $94^{\circ} \mathrm{C} / 3 \mathrm{~min} ; 30$ cycles of $94^{\circ} \mathrm{C} / 30 \mathrm{~s}, 55^{\circ} \mathrm{C} / 30$ $\mathrm{s}, 72^{\circ} \mathrm{C} / 2 \mathrm{~min}$; 1 cycle of $72^{\circ} \mathrm{C} / 10 \mathrm{~min}$. The PCR products were ligated into pGEM-T easy vector (Promega, USA), transformed into competent Escherichia coli DH5 $\alpha$ cells, plated onto LB-agar medium and incubated overnight at $37^{\circ} \mathrm{C}$. Positive clones containing the insert with the expected size were identified by colony PCR and 5 to 6 positive clones for each gene were picked and sequenced on an ABI PRISM 3730XL automated sequencer, using the BigDye terminator v4.10 kit (Applied Biosystems, USA).

To obtain the full-length cDNA sequence, $3^{\prime}$ RACE and 5' RACE were performed by nested PCR using gene-specific primers and universal primers (Table 1). The RT- and RACEPCR were conducted using the SMART RACE cDNA amplification kit (Clontech, Palo Alto, CA, USA) and Advantage 2 PCR kit (Clontech) following the manufacturer protocol. The PCR conditions for the $3^{\prime} \mathrm{RACE}$ was 1 cycle of $94^{\circ} \mathrm{C} / 3 \mathrm{~min} ; 30$ cycles of $94^{\circ} \mathrm{C} / 30 \mathrm{~s}, 52^{\circ} \mathrm{C} / 30$ $\mathrm{s}, 72^{\circ} \mathrm{C} / 2 \mathrm{~min}$; and 1 cycle of $72^{\circ} \mathrm{C} / 10 \mathrm{~min}$. Similarly, the PCR conditions for the $5^{\prime} \mathrm{RACE}$ was

Table 1. Oligonucleotide primers used to amplify the gcIL-12 p35 and gcIL-12 p40 genes.

\begin{tabular}{llllll}
\hline & \multicolumn{2}{c}{ gcIL-12 $\mathrm{p} 35$} & & \multicolumn{2}{c}{ gcIL-12 $\mathrm{p} 40$} \\
\cline { 2 - 2 } Primer & Sequence (5'-3') & & Sequence (5'-3') & Application \\
\hline F & TAGTTGTGGCTCTTGCGA & & TATCTGTGGAGAAGTCTAC & Short sequence verification \\
R & ACAACCCACTGAGGAAAC & & TTGGACAGCGAATCTCTT & Short sequence verification \\
3-race1 & TGATTCCTTACACTCACACAG & & GAGTCTGAACAGGGATGGC & 3'-RACE outer PCR \\
3-race2 & GAGAGTTTCCTCAGTGGGT & & TATTCAGAGGAGGTGTATCGC & 3'-RACE inner PCR \\
5-race1 & GCTCCAGACAACCCACTGAGGAAACT & & GAGGTAAGTCTCTGGCGAGTTCGGTTT & 5'-RACE outer PCR \\
5-race2 & CAGACGGACGCTGTGTGAGTGTAAGG & & CCAGTGTGTGGTTTAGGTAGGAGCCC & 5'-RACE inner PCR \\
RT-F & AGGCTCGGATGATTCCTTACA & & GGAGAAGTCTACGAAGGGCAA & Real-time PCR \\
RT-R & TCACACTGGGCTGGTAGGAG & & GTGTGTGGTTTAGGTAGGAGCC & Real-time PCR \\
Actin-F & GCTATGTGGCTCTTGACTTCG & & GCTATGTGGCTCTTGACTTCG & Real-time PCR (control) \\
Actin-R & CGCAAGACTCCATACCCAAG & & CGCAAGACTCCATACCCAAG & Real-time PCR (control) \\
\hline
\end{tabular}


5 cycles of $\left(94^{\circ} \mathrm{C} / 30 \mathrm{~s} ; 72^{\circ} \mathrm{C} / 3 \mathrm{~min}\right), 5$ cycles of $\left(94^{\circ} \mathrm{C} / 30 \mathrm{~s} ; 70^{\circ} \mathrm{C} / 30 \mathrm{~s} ; 72^{\circ} \mathrm{C} / 3 \mathrm{~min}\right)$, followed by 25 cycles $\left(94^{\circ} \mathrm{C} / 30 \mathrm{~s} ; 68^{\circ} \mathrm{C} / 30 \mathrm{~s} ; 72^{\circ} \mathrm{C} / 3 \mathrm{~min}\right)$. PCR products were cloned and sequenced as described above.

\section{Bioinformatic analysis}

The nucleotide and amino acid sequence identity searches were performed using the BLAST program (http://blast.ncbi.nlm.nih.gov/Blast.cgi). The open reading frames (ORF) of gcIL-12 p35 and p40 cDNA were predicted using the ORF Finder (http://www.ncbi.nlm.nih. gov/projects/gorf/), the presence of signal peptides were analyzed using the SignalP 4.1 server (http://www.cbs.dtu.dk/services/SignalP/) (Petersen et al., 2011), and putative domains and possible N-glycosylation sites were identified by PROSITE (http://www.expasy.org/prosite) (Falquet et al., 2002). The deduced amino acid sequence was analyzed with the Expert Protein Analysis System (http://www.expasy.org/). The EMBOSS Needle program (http://www.ebi. ac.uk/Tools/emboss/align/) was used to determine the identities between different sequences. Multiple sequence alignments were performed using the ClustalW 1.8 program (Jeanmougin et al., 1998). The phylogenetic tree was constructed based on the deduced full-length amino acid sequences using the neighbor-joining (NJ) algorithm in MEGA version 4.1, and analysis reliability was assessed by 1000 bootstrap replicates (Tamura et al., 2007).

\section{Real-time quantitative PCR analysis}

First-strand cDNA was synthesized from total RNA of each tissue using the PrimerScript first strand cDNA synthesis kit (TaKaRa, Japan), following the manufacturer instructions. Primers were designed based on the gcIL-12 p35 and p40 cDNA sequences obtained in this study (Table 1). Melting curve analysis was carried out to select the optimum primer pairs. A 10-fold dilution series of cDNA containing target gene fragments was used to construct standard curves and the amplification efficiency was measured from the regression slope of the standard curve. Only those primer pairs that showed a single peak in the melting curve analysis and displayed amplification efficiency close to the theoretical $100 \%$ were retained for further use. Amplification of grass carp $\beta$-actin mRNA was performed to confirm the steadystate level of expression of a housekeeping gene and provided as an internal control for the gcIL-12 p35 and p40 gene expression analysis. A PCR run using the same reaction mixture without template was conducted as a negative control. Real-time quantitative PCR (qPCR) was performed in triplicate for each sample with SYBR Premix EX Taq (TaKaRa, Japan) using a CFX96 real-time system (Bio-Rad, USA). The PCR parameters for both genes were as follows: $95^{\circ} \mathrm{C}$ for $3 \mathrm{~min}, 39$ cycles at $95^{\circ} \mathrm{C}$ for $5 \mathrm{~s}, 60^{\circ} \mathrm{C}$ for $30 \mathrm{~s}$, followed by a dissociation curve analysis of $5 \mathrm{~s}$ per step from $65^{\circ} \mathrm{C}$ to $95^{\circ} \mathrm{C}$. Relative expression analyses were based on the level of gcIL-12 p35 and p40 gene expression normalized against $\beta$-actin according to the $2^{-\Delta \Delta \mathrm{Ct}}$ method (Livak and Schmittgen, 2001).

Data from the qRT-PCR experiments were analyzed through one-way analysis of variance (ANOVA) using the SPSS (version 16.0) statistical software (SPSS Inc., Chicago, IL, USA). Differences between means were considered to be statistically significant at the $95 \%$ confidence level $(\mathrm{P}<0.05)$. All data are reported as means $\pm \mathrm{SE}$ (standard error). 


\section{RESULTS}

\section{Cloning and characterization of p35 subunit of grass carp IL-12}

Full-length gcIL-12 p35 cDNA consisted of 1021 nucleotides (nt) (GenBank accession number KF944667). It included a 31-nt 5' untranslated region (UTR), a 588-nt ORF, and a 402-nt 3' UTR with a canonical polyadenylation signal (AATAAA), $18 \mathrm{nt}$ upstream of the poly-A tail and 4 potential mRNA instability motifs (TATTTAT). The ORF encoded a 195-amino acid (aa) protein with a putative 16-aa signal peptide, which would generate a mature protein with calculated molecular mass of approximately $19.689 \mathrm{kDa}$ and theoretical isoelectric point (pI) of 6.56. The mature protein of gcIL-12 p35 contained 7 cysteine residues and 4 predicted $\mathrm{N}$-glycosylation sites $\left(\mathrm{N}^{38}, \mathrm{~N}^{59}, \mathrm{~N}^{84}\right.$, and $\mathrm{N}^{103}$ ) (Figure 1A). Grass carp $\mathrm{C}^{60}, \mathrm{C}^{77}, \mathrm{C}^{87}, \mathrm{C}^{100}$, and $\mathrm{C}^{168}$ are conserved in IL-12 p35 across all vertebrate species. $\mathrm{N}^{59} \mathrm{CT}$ is conserved across some teleost IL-12 p35 sequences. Despite the different levels of amino acid identities, most of the key residues involved in intrachain disulphide binding (corresponding to human $\mathrm{C}^{64}-\mathrm{C}^{196}$, and $\mathrm{C}^{85}-\mathrm{C}^{123}$ ) and heterodimerization with $\mathrm{p} 40$ (corresponding to human $\mathrm{C}^{96}, \mathrm{R}^{205}, \mathrm{~T}^{208}$, and $\mathrm{R}^{211}$ ) are conserved in gcIL-12 p35 (Figure 1A).

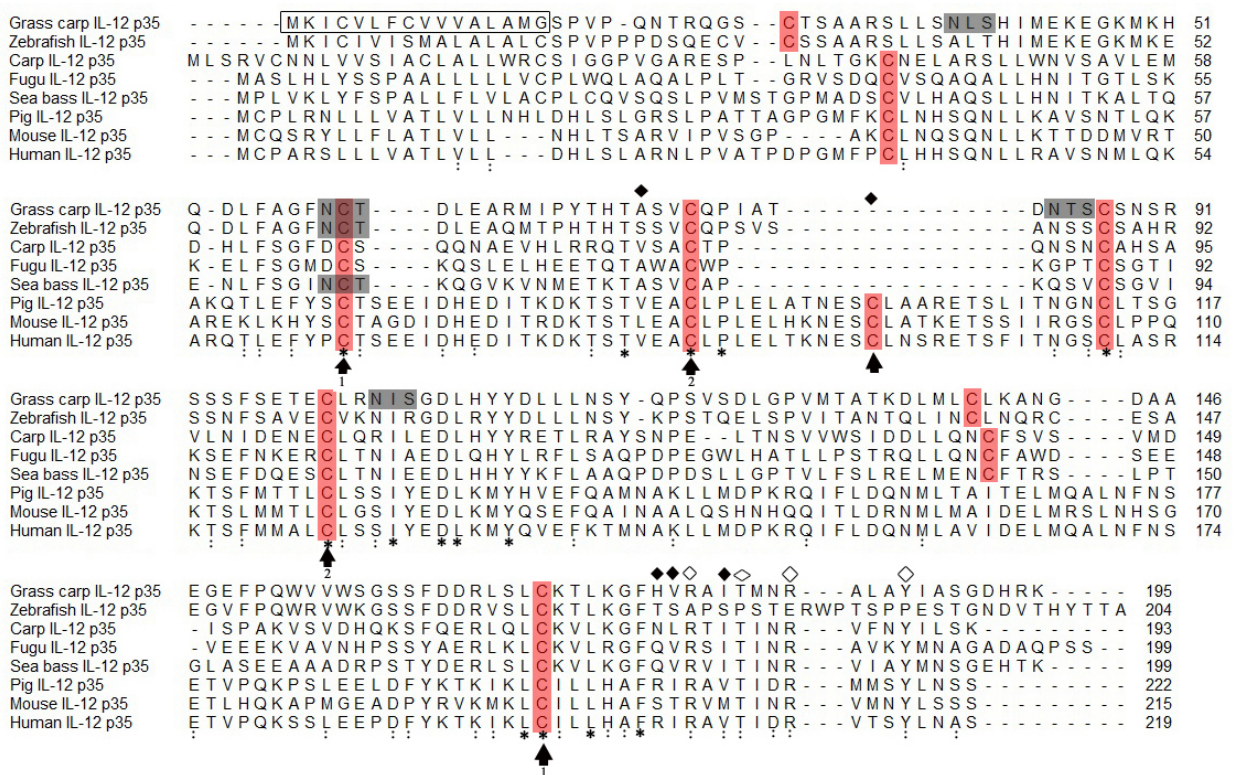

Figure 1A. Amino acid sequence alignment of IL-12 p35 from several teleost species using the ClustalW 1.8 program. Dashes indicate gaps introduced to optimize the similarity between sequences. Identical amino acids among all sequences are indicated by "asterisk" whereas those with high similarity are indicated by "colon". The predicted signal peptide is marked within a box. Conserved cysteine residues are shaded in red, while potential $\mathrm{N}$-glycosylation sites are shaded in black. Arrow without numbers indicates cysteine residues involved in the human IL-12 inter-chain disulphide bridge. Cysteine residues forming intra-chain disulphide bridges in human IL12 are indicated by closed, numbered arrows. Structurally critical (filled diamond) and important amino acids (open diamond) in human IL-12 p70 are shown above the alignments. The GenBank accession numbers of the sequences used for the alignment are as follows: Zebrafish NP_001007108.1; Carp CAE45012.1; Fugu NP_001094075.1; Sea bass ABD46706.1; Pig AGZ15484.1; Mouse NP_445842.1; Human NP_000873.1. 


\section{Cloning and characterization of p40 subunit of grass carp IL-12}

Full-length p40 cDNA consisted of 1642 nucleotides (nt) (GenBank accession number KF944668). It included a 326-nt 5' UTR, a 993-nt ORF, and a 323-nt 3' UTR with a canonical polyadenylation signal (AATAAA) $16 \mathrm{nt}$ upstream of the poly-A tail and two potential mRNA instability motifs (TATTTAT). The ORF encoded a 330-aa protein with a putative 17-aa signal peptide, which would generate a mature protein with calculated molecular mass of approximately $36.119 \mathrm{kDa}$ and theoretical $\mathrm{pI}$ of 8.99 . The mature protein of gcIL-12 p40 contained 13 cysteine residues and 7 predicted $\mathrm{N}$-glycosylation sites $\left(\mathrm{N}^{62}, \mathrm{~N}^{103}, \mathrm{~N}^{164}, \mathrm{~N}^{194}, \mathrm{~N}^{224}, \mathrm{~N}^{229}\right.$, and $\mathrm{N}^{301}$ ). Grass carp $\mathrm{C}^{94}, \mathrm{C}^{128}, \mathrm{C}^{139}, \mathrm{C}^{178}$, and $\mathrm{C}^{184}$ are conserved across all vertebrate species, $\mathrm{C}^{167}$, $\mathrm{C}^{265}, \mathrm{C}^{267}, \mathrm{C}^{292}$, and $\mathrm{C}^{300}$ are conserved within all teleost while $\mathrm{C}^{54}$ and $\mathrm{C}^{259}$ are conserved within freshwater teleost IL-12 p40 (Figure 1B). $\mathrm{N}^{62} \mathrm{IT}, \mathrm{N}^{164} \mathrm{IS}, \mathrm{N}^{194} \mathrm{LT}$, and $\mathrm{N}^{301} \mathrm{SS}$ are conserved among freshwater fish IL-12 p40 sequences, whereas $\mathrm{N}^{124} \mathrm{IS}$ is unique to grass carp. The gcIL$12 \mathrm{p} 40$ contained an N-terminal immunoglobulin (Ig) domain and two fibronectin type-III domains (Figure 1B). The first fibronectin domain conserves 4 typical cysteines $\left(\mathrm{C}^{128}, \mathrm{C}^{139}, \mathrm{C}^{178}\right.$, and $\mathrm{C}^{184}$ ) and the second contains the well-conserved WSXWS motif. The family signature of the long hematopoietin receptor soluble $\alpha$-chains is also partially conserved on gcIL-12 p40.

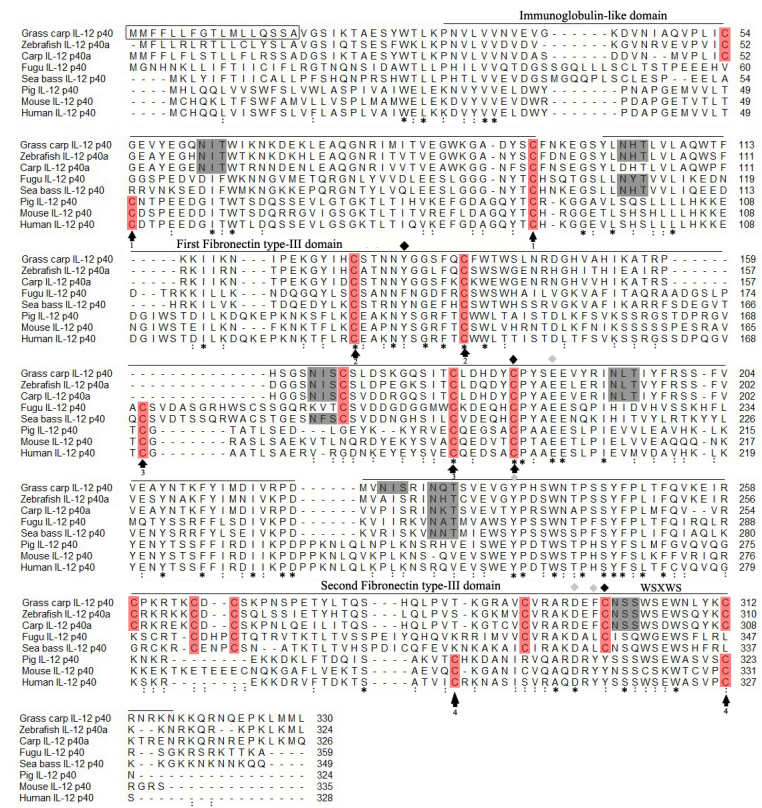

Figure 1B. Amino acid sequence alignment of IL-12 p40 from several teleost species using the ClustalW 1.8 program. Dashes indicate gaps introduced to optimize the similarity between sequences. Identical amino acids among all sequences are indicated by "asterisk" whereas those with high similarity are indicated by "colon". The predicted signal peptide is marked within a box. Conserved cysteine residues are shaded in red, while potential $\mathrm{N}$-glycosylation sites are shaded in black. Arrow without numbers indicates the cysteine residues involved in the human IL-12 inter-chain disulphide bridge. Cysteine residues forming intra-chain disulphide bridges in human IL12 are indicated by closed numbered arrows. Structurally critical (filled diamond) and important amino acids (open diamond) in human IL-12 p70 are shown above the alignments. The GenBank accession numbers of the sequences used for the alignment are as follows: Zebrafish NP_001007109.1; Carp CAF18555.1; Fugu BAC81425.1; Sea bass ABD46708.1; Pig NP_999178.1; Mouse NP_072133.1; Human NP_002178.2. 


\section{Multiple alignment and phylogenetic analysis of gcIL-12}

To study the evolutionary relationship of gcIL-12 p35 and p40 proteins with those from other known vertebrates, amino acid sequences corresponding to representative orthologs from several species were aligned with gcIL-12 p35 and p40 using the ClustalW software. The deduced amino acid sequence analysis showed that gcIL-12 p35 is more similar to zebrafish orthologues with $65.1 \%$ similarity and $53.2 \%$ identity; it showed moderate identity with p35 from other fish species such as sea bass (33.0\%), common carp (31.7\%), and fugu (28.7\%), and low identity with mammalian p35 such as human (20.8\%). Similarly, the gcIL$12 \mathrm{p} 40$ showed the highest identity with common carp and zebrafish $\mathrm{p} 40 \mathrm{a}(77.3 \%$ and $73.6 \%$, respectively), moderate identity with marine fishes p40 such as sea bass (37.6\%) and fugu $(32.4 \%)$, and the lowest identity with mammalian p40 such as human $(27.1 \%)$.

The phylogenetic tree showed two distinct clades for each of the p35 and p40 groups, with clade containing all teleost fish sequences and the other with mammalian and avian sequences (Figure 2). The gcIL-12 p35 was clustered with teleost orthologs with the highest

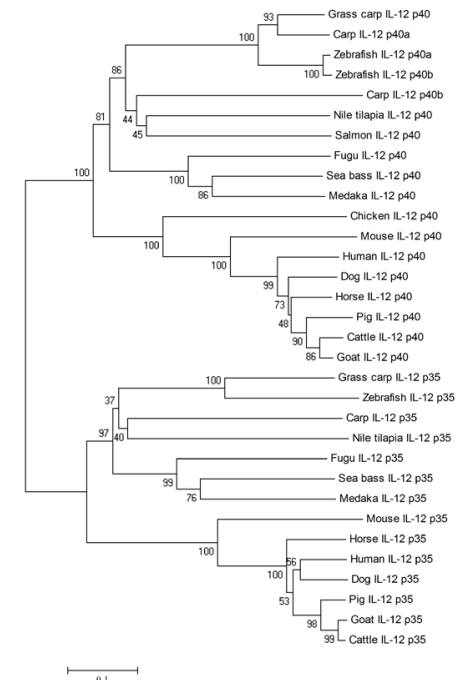

Figure 2. Phylogenetic tree showing the relationship between gcIL-12 p35 and p40 amino acid sequences and other identified IL-12 p35 and p40 sequences. The tree was constructed using the neighbor-joining method (Tree Top Program) with ClustalW and MEGA 4.1 packages and was bootstrapped 1000 times. The GenBank accession numbers of the sequences used for the phylogenetic analysis are as follows: Zebrafish (Danio rerio) IL12 p35 NP_001007108.1; Sea bass (Dicentrarchus labrax) IL-12 p35 ABD46706.1; Carp (Cyprinus carpio) IL-12 p35 CAE45012.1; Fugu (Takifugu rubripes) IL-12 p35 NP_001094075.1; Medaka (Oryzias latipes) IL-12 p35 XP_004079386.1; Goat (Capra hircus) IL-12 p35 AAT72317̄.1; Pig (Sus scrofa) IL-12 p35 AGZ15484.1; Human (Homo sapiens) IL-12 p35 NP_000873.1; Nile tilapia (Oreochromis niloticus) IL-12 p35 XP_003454873.2; Dog (Canis lupus familiaris) IL-12 p̈35 NP_001003293.1; Horse (Equus caballus) IL-12 p35 NP_001075980.1; Mouse (Rattus norvegicus) IL-12 p35 NP_445842.1; Cattle (Bos taurus) IL-12 p35 NP_776780.1; Zebrafish (Danio rerio) IL-12 p40a NP 001007109.1; IL-12 p40b CAJ44438.1; Sea bass (Dicentrarchus labrax) IL-12 p40 ABD46708.1; Carp (Cyprinus carpio) IL-12 p40a CAF18555.1; IL-12 p40b CAF32323.1; Fugu (Takifugu rubripes) IL-12 p40 BAC81425.1; Medaka (Oryzias latipes) IL-12 p40 XP_004073574.1; Salmon (Salmo salar) IL-12 p40 ACI69563.1; Goat (Capra hircus) IL-12 p p40 NP_001272629.1; Pig (Sus scrofa) IL-12 p40 NP_999178.1; Human (Homo sapiens) IL-12 p40 NP_002178.2; Nile tilapia (Oreochromis niloticus) IL-12 p40 XP_003447003.1; Dog (Canis lupus familiaris) IL-12 $\overline{\mathrm{p}} 40$ NP_001003292.1; Horse (Equus caballus) IL-12 p40 NP_0001075985.1; Mouse (Rattus norvegicus) IL-12 p40 NP_072133.1; Cattle (Bos taurus) IL-12 p40 NP_776781.1; C̄hicken (Gallus gallus) IL-12 p40 AAP86972.1. 
similarity to zebrafish. The gcIL-12 p40 was also clustered with teleost orthologs proteins with the highest similarity to carp p40a.

\section{Expression of gcIL-12 mRNAs in normal tissues}

Expression of gcIL-12 p35 and p40 transcripts in tissue was examined by qRT-PCR analysis normalized against $\beta$-actin levels. The tissues included the gill, brain, heart, blood, fin, skin, liver, trunk kidney, head kidney, spleen, and intestine. Expression was determined as fold-increased $2^{-\triangle \Delta \mathrm{Ct}}$ levels relative to the tissue with lowest expression set at 1 . As shown in Figure 3A-B, both gcIL-12 subunits were expressed in a wide range of tissues, although obvious differences were found. The highest expression of gcIL-12 p35 was observed in the brain (6.1-fold), followed by gill (4.6-fold), and fin (3.3-fold); expression was very low in the trunk kidney (the calibrator), intestine (1.3-fold), and spleen (1.9-fold) (Figure 3A). The gcIL-12 p40 mRNA was expressed at the highest level in the heart (65.6-fold), spleen (51.7-fold), and blood (48.4-fold), followed by head kidney (41.4-fold), gill (40.7-fold), and intestine (33.7fold) $(\mathrm{P}<0.05)$; expression was very low in the liver (the calibrator), skin (2.4-fold), and fin (2.8-fold) (Figure 3B).
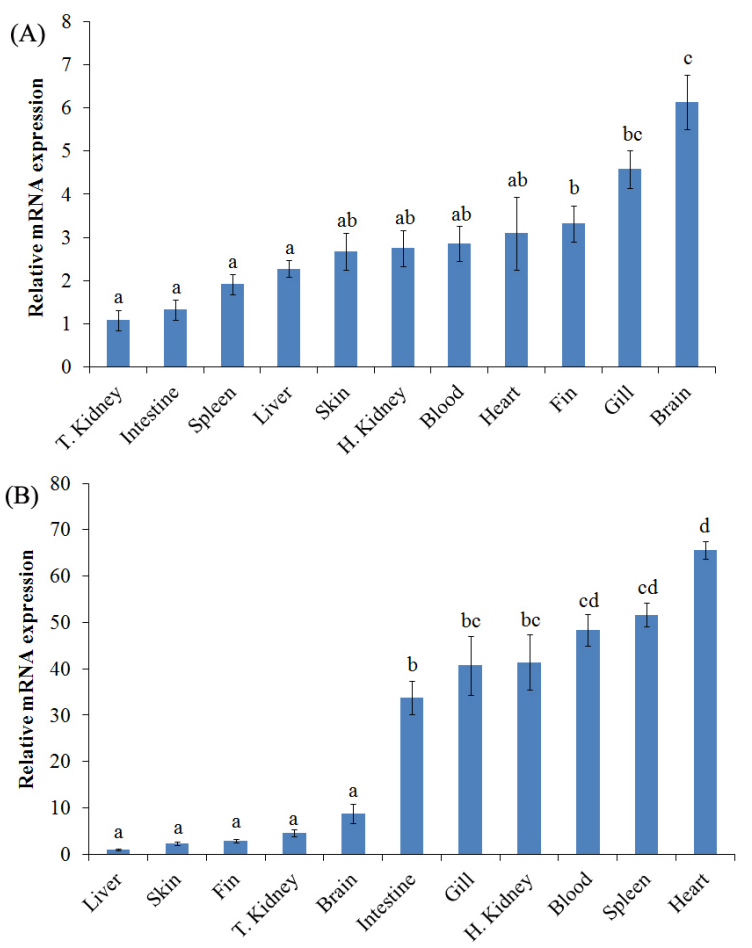

Figure 3. Tissue expression profiles of gcIL-12 p35 (A) and gcIL-12 p40 (B) genes. Amounts of mRNA levels measured by quantitative real-time PCR (qRT-PCR) are indicated. The relative expression of each gene transcript was calculated based on the standard curve and was normalized to the B-actin mRNA level. Data from qRT-PCR are reported as means $\pm \mathrm{SE}$ (standard error). Results shown are the mean of six individual RNA samples of fish $(\mathrm{N}=6)$. The data with different superscript letters indicate significantly different at $\mathrm{P}<0.05$. 


\section{Expression of gcIL-12 mRNAs in different tissues after exposure to $A$. hydrophila}

The expression pattern of gcIL-12 p35 and p40 mRNAs were assessed by qRT-PCR in samples from the gill, spleen, head kidney, and intestine of grass carp at $4 \mathrm{~h}, 1$ day, 3 days, and 7 days post injection of $A$. hydrophila. Relative expression was determined as fold changes relative to the PBS-injected control samples. After infection with A. hydrophila, both genes were upregulated or downregulated in a tissue-specific and time-specific manner. However, in all tissues, the expression of $\mathrm{p} 35$ closely paralleled that of $\mathrm{p} 40$. Among all tested tissues, the highest upregulation of both p35 and p40 mRNA was observed in the gill (16.9- and 26.9-fold, respectively) at 7 days post challenge. In the gill and spleen, expression of both p35 and p40 was significantly upregulated at 1 -day post challenge, downregulated at 3 days post challenge, and again highly upregulated at 7 days post challenge $(\mathrm{P}<0.05)$ (Figure 4A-B). In the head kidney, expression of both, p35 and p40 was gradually upregulated from $4 \mathrm{~h}$ to 1 day post challenge, returned to normal level at 3 days and 7 days post challenge (Figure 4C). In the intestine, p35 expression was significantly upregulated at $4 \mathrm{~h}$ post challenge, which gradually decreased and was downregulated at 3 days, and again highly upregulated at 7 days post challenge $(\mathrm{P}<0.05)$. However, the expression of $\mathrm{p} 40$ was almost similar to that of the control at all time points (Figure 4D).
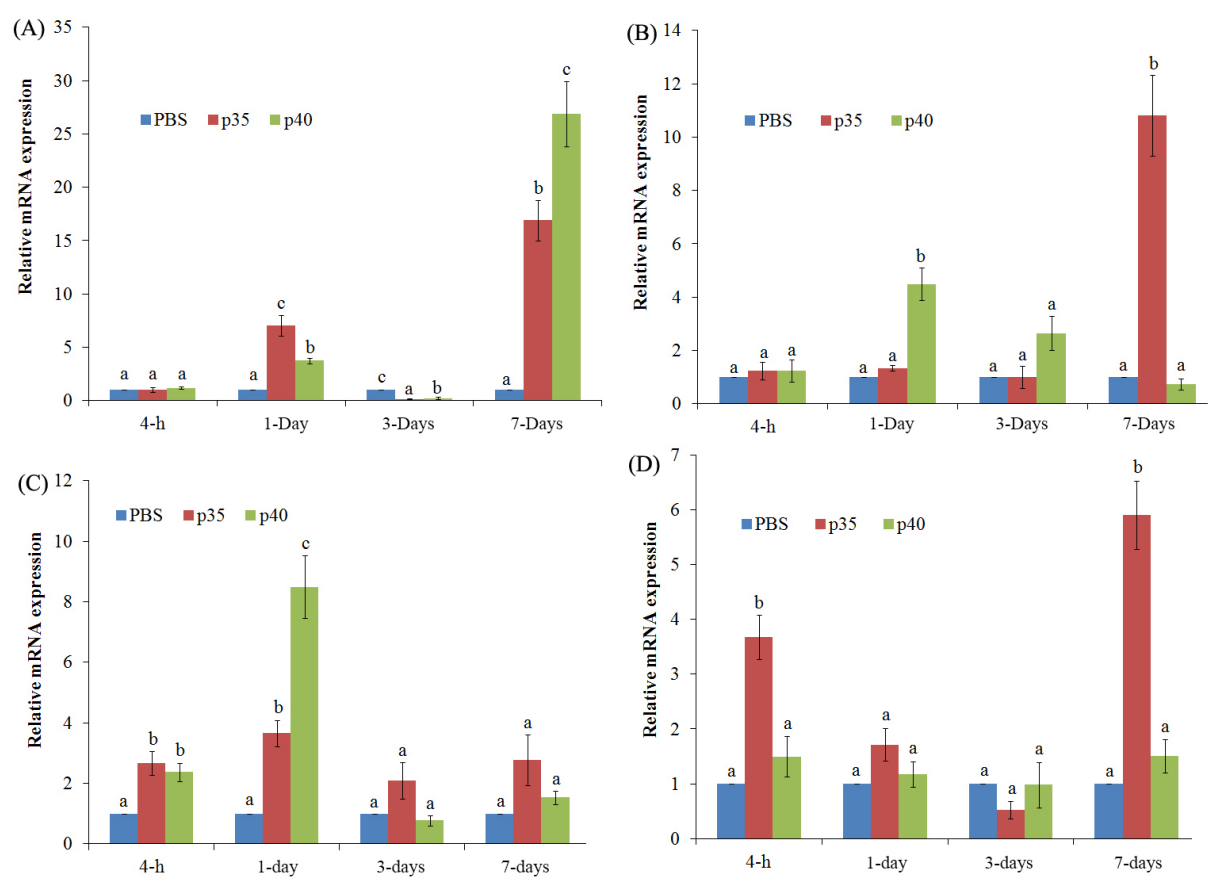

Figure 4. Quantitative expression profiles of gcIL-12 p35 and p40 genes in the gill (A), spleen (B), head kidney (C), and intestine (D) at different time points post challenge with Aeromonas hydrophila. Control samples were injected with PBS. The relative expression of each gene transcript is shown as fold changes as compared to PBSinjected control samples, normalized to changes in expression levels of $\beta$-actin mRNA. Data from qRT-PCR are reported as means \pm SE. Results shown are the mean of six individual RNA samples of fish $(\mathrm{N}=6)$. The data with different superscript letters within the same tissue group indicate significant differences at $\mathrm{P}<0.05$. 


\section{Expression of gcIL-12 mRNA in different tissues after exposure to Aquareovirus}

The expression patterns of gcIL-12 p35 and p40 mRNA were investigated in the gill, spleen, head kidney, and intestine of grass carp at $4 \mathrm{~h}, 1$ day, 3 days, and 7 days post injection with Aquareovirus, using qRT-PCR. Relative expression was determined as fold changes relative to the PBS-injected control samples. Similar to results with A. hydrophila infection, the expression of both genes was upregulated and downregulated in a tissue- and time-specific manner after Aquareovirus infection (Figure 5A-D). In the gill, p40 expression was highly upregulated at 1 day post challenge (6.9-fold), and then downregulated at 3 days and 7 days post challenge $(\mathrm{P}<0.05)$; whereas no upregulation was observed in $\mathrm{p} 35$ (Figure $5 \mathrm{~A})$. In the spleen, both, p35 and p40 expression was significantly upregulated at $4 \mathrm{~h}$ (5.1- and 2.0-fold, respectively), returned to normal level at 1 day, upregulated again at 3 days (1.8- and 5.1-fold, respectively), and then returned to normal level at 7 days post challenge (Figure 5B). In the head kidney, both, p35 and p40 expression was significantly upregulated at $4 \mathrm{~h}$ post challenge (4.2- and 2.9-fold, respectively), returned to normal level at 1 day, and again upregulated at 7 days post challenge (2.0- and 1.6-fold, respectively) (Figure 5C). In the intestine, both, p35 and $\mathrm{p} 40$ expression was highly upregulated at $4 \mathrm{~h}(4.6$ - and 4.8 -fold, respectively) $(\mathrm{P}<0.05)$, gradually decreased at 1 day post challenge (2.7- and 2.2-fold, respectively) and returned to normal levels at 3 and 7 days post challenge (Figure 5D).
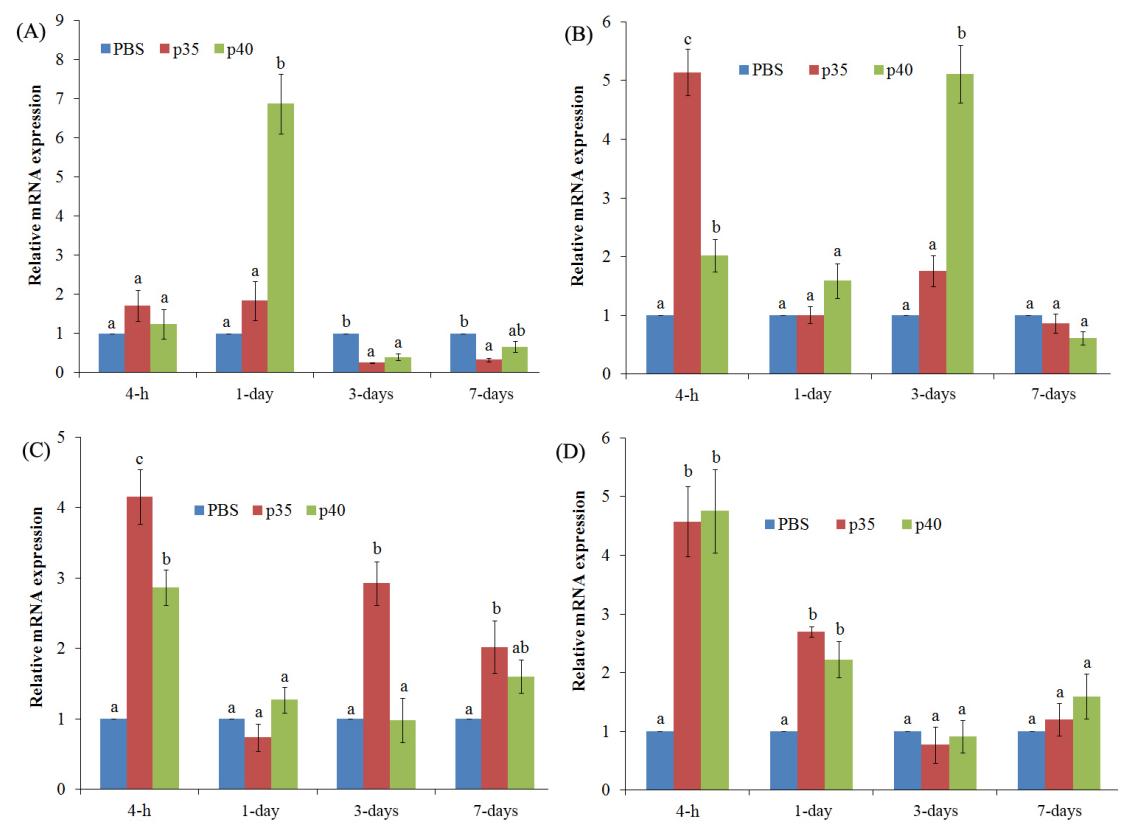

Figure 5. Quantitative expression profiles of gcIL-12 p35 and p40 genes in the gill (A), spleen (B), head kidney (C), and intestine (D) at different time points post challenge with Aquareovirus (GCRV-JX01). Control samples were injected with PBS. The relative expression of each gene transcript is shown as fold changes as compared to PBS-injected control samples, normalized to changes in expression levels of $\beta$-actin mRNA. Data from qRT-PCR are reported as means $\pm \mathrm{SE}$. Results shown are the mean of six individual RNA samples of fish $(\mathrm{N}=6)$. The data with different superscript letters within the same tissue group indicate significant difference at $\mathrm{P}<0.05$. 


\section{DISCUSSION}

IL-12 is the primary pro-inflammatory cytokine which plays a key role in the regulation of adaptive immunity in vertebrates (Secombes et al., 2011). In this study, the involvement of IL-12 subunits, p35 and p40 in the anti-bacterial and anti-viral immune responses of grass carp was demonstrated by examining the expression profiles of these genes in the immune tissues following infection by Aeromonas hydrophila and Aquareovirus. The p35 and p40 subunits of IL-12 from teleost fish were first isolated by fugu genome analysis (Yoshiura et al., 2003), and then cloned from a few other fish species and characterized. Although previous studies have described cloning and expression of IL-12 subunits, p35 and p40, the response of these genes after A. hydrophila and Aquareovirus infection in fish are not yet understood. This is the first report on the identification and characterization of IL-12 subunit cDNA and genes in grass carp and an analysis of their expression after infection by A. hydrophila and Aquareovirus. The gcIL-12 subunits, p35 and p40 identified in this study, are IL-12 orthologues, which is clearly supported by the conservation of important structural residues, phylogenetic analysis, and gene organization. Our results show that many of the structural residues are conserved in freshwater fish, distinct from marine fish and mammals. In the common carp, a single p35 gene and 3 distinct $\mathrm{p} 40$ genes, named p40a, p40b, and p40c, have been identified (Huising et al., 2006). Similarly, a single p35 gene and 2 distinct p40 genes, named p40a and p40b, have been identified in zebrafish. Among p40 from carp and zebrafish, the gcIL-12 p40 is identical to carp p40a and zebrafish p40a, indicating that the gcIL-12 p40 might be a p40a orthologue. In this study, we were unable to detect the presence of $\mathrm{p} 40 \mathrm{~b}$ and $\mathrm{p} 40 \mathrm{c}$ in grass carp, which is similar to the report by Tsai et al (2014) in grouper fish.

The gcIL-12 p35 consists of an ORF of 588 bases with a coding capacity of 195 amino acids. Comparison of gcIL-12 p35 with other vertebrates showed that many of the structurally and functionally important features are conserved in gcIL-12 p35, such as a putative signal peptide, 7 cysteine residues, and 4 predicted N-glycosylation sites. Amino acid sequence comparison and phylogenetic analysis revealed that gcIL-12 p35 is more identical to zebrafish than other teleosts. Some of the important structural features are conserved only in grass carp and zebrafish. Similarly, the ORF cDNA of gcIL-12 p40 consists of 993 bases encoding a polypeptide of 330 amino acids. Amino acid sequence comparison and phylogenetic analysis revealed that gcIL-12 p40 is highly identical to carp and zebrafish p40a. In vertebrates, the tertiary structure of IL-12 p40 is divided into 3 structural domains (D1, D2, and D3) that seem to be conserved in gcIL-12 p40 (Yoon et al., 2000; Nascimento et al., 2007). The gcIL-12 p40 retains the $\mathrm{N}$-terminal Ig-like region with the two conserved cysteines known to form an intrachain disulfide bond within the human p40 D1 domain. In addition, gcIL-12 p40 conserves the 4 cysteine residues that form 2 disulphide bonds in the human p40 D2 domain. The WSXWS signature of Il-12 p40 is an important structural feature in efficient receptor folding and secretion for hemopoietin receptor family (de Vos et al., 1992), which is also conserved in D3 domain of gcIL-12 p40.

Both, gcIL-12 p35 and p40 are broadly expressed; however, tissue-specific expression levels of these two molecules are highly different. The brain expresses the highest level of gcIL-12 p35, suggesting its role in the central nervous system. Although higher expression of gcIL-12 p35 was detected in some immune tissues such as in gills; intermediate to low degree of expression is observed in most of the immune tissues including the head kidney, skin, spleen, liver, as well as intestine, and this suggests that a different role of IL-12 p35 in the 
fish. Thus, it is possible that IL-12 p35 might be involved in the sensory control mechanism for immune regulation. In this study, the heart and blood expressed the highest level of IL-12 p40, suggesting its role in fish circulation. IL-12 p40 expression was also highly detected in immune tissues including the spleen, head kidney, gills, as well as intestine, and this suggests that it has an important role in the fish immune system. In mammals, IL-12 is primarily produced by phagocytic cells in response to many infectious or inflammatory stimuli (Trinchieri, 2003). However, expression of IL-12 p35 and p40 mRNAs in non-lymphoid tissues of grass carp in this study is difficult to interpret at present. Further analysis is required to clarify which cell types express gcIL-12 p35 and p40 mRNA in lymphoid and non-lymphoid tissues. Almost similar expression patterns of IL-12 p35 and p40 were reported in different tissues in fugu (Yoshiura et al., 2003). In carp, the expression of IL-12 p35 was closely paralleled with that of IL-12 p40a in all organs (Huising et al., 2006). In contrast, we found that IL-12 p35 is produced at much lower levels than that of IL-12 p40 in grass carp.

The biological role of IL-12 in freshwater fish is still unclear. Some previous studies demonstrated that the teleost IL-12 p35 and p40 mRNAs are stimulated in some tissues by viral (Huising et al., 2006) and bacterial infections (Nascimento et al., 2007). Recently, Tsai et al. (2014) have exhibited the cytokine and chemokine activities of IL-12 in grouper fish. On the contrary, IL-12 p40 was reported to be unresponsive to bacterial and viral stimulation in fugu (Yoshiura et al., 2003) as well as in spring viraemia of carp virus (SVCV) stimulation in carp (Forlenza et al., 2008). To elucidate the anti-bacterial anti-viral properties of IL-12 p35 and p40 in grass carp, a freshwater species, we performed basic bacterial and viral challenge experiments using A. hydrophila and Aquareovirus, respectively. Our results demonstrated that expression levels of gcIL-12 p35 and p40 mRNAs show tissue- and time-specific changes following challenge. The gcIL-12 p35 gene was significantly upregulated in spleen, head kidney, and intestine within 4 hours of Aquareovirus injection (4.2- to 5.2-fold), whereas it was unresponsive in gill tissues until 7-d post injection. Expression of gcIL-12 p35 gene was also induced in all tested tissues after A. hydrophila injection (3.7- to 16.9-fold). However, upregulation was detected much earlier in head kidney and intestine (4-h post injection) than in spleen and gills (7-d post injection). These evidences indicate that gcIL-12 p35 is involved in the early stage of anti-viral and anti-bacterial innate immune reactions. Similarly, the gcIL-12 p40 gene was significantly upregulated in spleen, head kidney, intestine and gills after Aquareovirus injection. It was also significantly upregulated in spleen, head kidney and gills after $A$. hydrophila injection, whereas not stimulated in intestine. In gills, the increased expression of p40 was only detected at late stage (7-d post injection). Interestingly, both p35 and p40 were significantly down-regulated at 3-d post injection of $A$. hydrophila and Aquareovirus. Although the clear mechanism of such time- and tissue-dependent upregulation or down-regulation of IL-12 after infection is unknown, it suggests that IL-12 expression is under strict control. Whether the decreased expression is the result of a decrease in transcription or increase in mRNA decay will need to be investigated further.

It has been reported that production of both $\mathrm{p} 35$ and p 40 subunits in the same cell are required to obtain the biologically active, heterodimeric IL-12 (D'Andrea et al., 1992). In this study, p35 expression was induced concomitantly with p40 gene expression in spleen, head kidney, and intestine tissues during viral infection. However, during bacterial infection, p35 expression was induced concomitantly with $\mathrm{p} 40$ gene expression only in gill and head kidney, but not in spleen and intestine. This indicates that, in some tissues, in vivo is the association of p35 with p40 which might lead to the formation of a functional IL-12(p35p40) heterodimeric 
molecule. However, the role of independent expressions of p35 and p40 in some tissues after infection is not clear. In the present study, both subunits of IL-12 have showed combine response against both bacterial and viral infections in head kidney, indicating that head kidney is the active site of action against bacterial and viral pathogens. Thus, head kidney might be an important tissue for further immunological study of IL-12.

In summary, we have cloned the full-length cDNA of IL-12 p35 and p40 subunits from grass carp, analyzed the structure of protein, examined their phylogenetic relationships, and demonstrated the expression profiles in normal tissues and after infection with $A$. hydrophila and Aquareovirus. The expression of gcIL-12 subunits, p35 and p40 in normal tissues suggest that they have roles in the nervous, circulation, and immune systems. The significant induction of gcIL-12 p35 and p40 by A. hydrophila and Aquareovirus infection confirms their role in anti-bacterial anti-viral immune processes in grass carp. Future studies will involve the production of recombinant IL-12 protein and the direct study of its immunological role in grass carp.

\section{ACKNOWLEDGMENTS}

Research supported by the Earmarked Fund for China Agriculture Research System (\#CARS-46-04), the National Key Technology R\&D Program (\#2012BAD26B02), the Agricultural Seed Development Program of Shanghai City (\#2012NY10), the Shanghai Ocean University Doctoral Research Foundation, the Shanghai Universities First-class Disciplines Project of Fisheries, and the Special Funding for the Development of Science and Technology of Shanghai Ocean University.

\section{REFERENCES}

Adorini L (1999). Interleukin-12, a key cytokine in Th1-mediated autoimmune diseases. Cell. Mol. Life Sci. 55: 16101625.

D'Andrea A, Rengaraju M, Valiante NM, Chehimi J, et al. (1992). Production of natural killer cell stimulatory factor (interleukin 12) by peripheral blood mononuclear cells. J. Exp. Med. 176: 1387-1398.

de Vos AM, Ultsch M and Kossiakoff AA (1992). Human growth hormone and extracellular domain of its receptor: crystal structure of the complex. Science 255: 306-312.

Ebner S, Ratzinger G, Krosbacher B, Schmuth M, et al. (2001). Production of IL-12 by human monocyte-derived dendritic cells is optimal when the stimulus is given at the onset of maturation, and is further enhanced by IL-4. J. Immunol. 166: 633-641.

Falquet L, Pagni M, Bucher P, Hulo N, et al. (2002). The PROSITE database, its status in 2002. Nucleic Acids Res. 30: 235-238.

FAO Fisheries and Aquaculture Department (2012). The state of world fisheries and aquaculture 2012, Rome.

Forlenza M, de Carvalho Dias JD, Vesely T, Pokorová D, et al. (2008). Transcription of signal-3 cytokines, IL-12 and IFN alpha beta, coincides with the timing of CD8 alpha beta upregulation during viral infection of common carp (Cyprinus carpio L). Mol. Immunol. 45: 1531-1547.

Gaucher D and Chadee K (2001). Molecular cloning of gerbil interleukin 12 and its expression as a bioactive single-chain protein. Cytokine 14: 177-183.

Gubler U, Chua AO, Schoenhaut DS, Dwyer CM, et al. (1991). Coexpression of two distinct genes is required to generate secreted bioactive cytotoxic lymphocyte maturation factor. Proc. Natl. Acad. Sci. U.S.A. 88: 4143-4147.

Huising MO, van Schijndel JE, Kruiswijk CP, Nabuurs SB, et al. (2006). The presence of multiple and differentially regulated interleukin-12p40 genes in bony fishes signifies an expansion of the vertebrate heterodimeric cytokine family. Mol. Immunol. 43: 1519-1533.

Jang S, Liu H, Su J, Dong F, et al. (2010). Construction and characterization of two bacterial artificial chromosome libraries of grass carp. Mar. Biotechnol. 12: 261-266. 
Jeanmougin F, Thompson JD, Gouy M, Higgins DG, et al. (1998). Multiple sequence alignment with Clustal X. Trends Biochem. Sci. 23: 403-405.

Jiang Y (2009). Hemorrhagic disease of grass carp: Status of outbreaks, diagnosis, surveillance, and research. Israel. J. Aqua. - Bamidgeh 61: 188-197

Kobayashi M, Fitz L, Ryan M, Hewick RM, et al. (1989). Identification and purification of natural killer cell stimulatory factor (NKSF), a cytokine with multiple biologic effects on human lymphocytes. J. Exp. Med. 170: 827-845.

Ling P, Gately MK, Gubler U, Stern AS, et al. (1995). Human IL-12 p40 homodimer binds to the IL-12 receptor but does not mediate biologic activity. J. Immunol. 154: 116-127.

Liu F, Wang D, Fu J, Sun G, et al. (2010). Identification of immune-relevant genes by expressed sequence tag analysis of head kidney from grass carp (Ctenopharyngodon idella). Comp. Biochem. Physiol. Part D Genomics Proteomics 5: 116-123.

Livak KJ and Schmittgen TD (2001). Analysis of relative gene expression data using real-time quantitative PCR and the 2[-Delta Delta C(T)] Method. Methods 25: 402-408.

Nascimento DS, do Vale A, Tomás AM, Zou J, et al. (2007). Cloning, promoter analysis and expression in response to bacterial exposure of sea bass (Dicentrarchus labrax L.) interleukin-12 p40 and p35 subunits. Mol. Immunol. 44: 2277-2291.

Pandit NP, Shen Y, Wang W, Chen Y, et al. (2013). Identification of TNF13b (BAFF) gene from grass carp (Ctenopharyngodon idella) and its immune response to bacteria and virus. Dev. Comp. Immunol. 39: 460-464.

Petersen TN, Brunak S, von Heijne G and Nielsen H(2011). SignalP 4.0: discriminating signal peptides from transmembrane regions. Nat. Methods 8: 785-786.

Secombes CJ, Wang T and Bird S (2011). The interleukins of fish. Dev. Comp. Immunol. 35: 1336-1345.

Staerk J and Constantinescu SN (2012). The JAK-STAT pathway and hematopoietic stem cells from the JAK2 V617F perspective. JAKSTAT 1: 184-190.

Tamura K, Dudley J, Nei M and Kumar S (2007). MEGA4: molecular evolutionary genetics analysis (MEGA) software version 4.0. Mol. Biol. Evol. 24: 1596-1599.

Trinchieri G (2003). Interleukin-12 and the regulation of innate resistance and adaptive immunity. Nat. Rev. Immunol. 3: 133-146.

Tsai JL, Priya TA, Hu KY, Yan HY, et al. (2014). Grouper interleukin-12, linked by an ancient disulfide-bond architecture, exhibits cytokine and chemokine activities. Fish Shellfish Immunol. 36:27-37.

Vignali DA and Kuchroo VK (2012). IL-12 family cytokines: immunological playmakers. Nat. Immunol. 13: 722-728.

Vivekanandhan G, Savithamani K, Hatha AA and Lakshmanaperumalsamy P (2002). Antibiotic resistance of Aeromonas hydrophila isolated from marketed fish and prawn of South India. Int. J. Food Microbiol. 76: 165-168.

Wang Q, Zeng W, Liu C, Zhang C, et al. (2012). Complete genome sequence of a reovirus isolated from grass carp, indicating different genotypes of GCRV in China. J. Virol. 86: 12466.

Yoon C, Johnston SC, Tang J, Stahl M, et al. (2000). Charged residues dominate a unique interlocking topography in the heterodimeric cytokine interleukin-12. EMBO J. 19: 3530-3541.

Yoshiura Y, Kiryu I, Fujiwara A, Suetake H, et al. (2003). Identification and characterization of Fugu orthologues of mammalian interleukin-12 subunits. Immunogenetics 55: 296-306. 